\title{
»N2B-Patch« - Nose-to-Brain Delivery of an Active Pharmaceutical Ingredient via the Olfactory Region
}

\author{
Carmen Gruber-Traub* and Jenny Ullrich \\ Fraunhofer Institute for Interfacial Engineering and Biotechnology IGB, Stuttgart, Germany
}

Received: May 04, 2018; Published: 眥 May 10, 2018

*Corresponding author: Dr. Carmen Gruber-Traub, Fraunhofer Institute for Interfacial Engineering and Biotechnology IGB, Nobelstrasse 12, 70569 Stuttgart, Germany

\begin{abstract}
Within the EU funded project »N2B-patch« eleven partners from eight countries aim to develop an innovative technology for the nose-to-brain delivery of an active pharmaceutical ingredient via the olfactory region for the regenerative treatment of multiple sclerosis using novel multi-functional biomaterials combined with a medical device. The four year EU-funded research involves an innovative method of bypassing the blood-brain barrier by delivering a potential multiple sclerosis drug with a special medical device via the nose directly to the brain.
\end{abstract}

Keywords: nose-to-brain, multiple sclerosis, multi-functional biomaterial, drug delivery, API-loaded particles

\section{Short Communication}

The World Health Organisation (WHO) estimates that more than one billion people worldwide are suffering from neurological disorders [1]. The therapy of central nervous system (CNS) diseases like multiple sclerosis (MS) is still an unmet medical need due to the low CNS bioavailability of innovative drugs like antibodies. Hence, a safe and efficient drug delivery platform technology for CNS active molecules is crucial and therefore needed.

MS is one of the most common long-term conditions affecting the CNS and the main cause of non-traumatic neurological disability in young adults. MS is a neurodegenerative disease, a group of conditions that include Parkinson's and Alzheimer's. It affects more than 700,000 people in Europe. MS is an inflammatory, demyelinating disease and it is estimated to affect 2.3 million people worldwide; prevalence is particularly high in Europe and North America, with rates over 100 cases per 100,000 [2]. Current standard therapies of MS include anti-inflammatory and symptomatic treatments. Although, relapse occurrence and duration are reduced by means of these therapies, no regeneration is achieved and there are no treatments for the progressive forms of MS. Therefore, MS is still a degenerative disease that leads to disability of patients in their most productive years. A key factor in the development of functional ingredients or active substances is their transport to their target location. Pharmaceutical active ingredients (APIs) are normally distributed via the blood, either directly by injection into the bloodstream or indirectly, for example through the digestive tract after oral administration. However, in many diseases, for example of the CNS, it is of decisive importance to transport the API as efficiently as possible to the required target site. An example of this is the treatment of MS, where the pharmaceutical agents have to produce their effect above all in the CNS. This is especially difficult to achieve in the usual way via the blood due to special protective mechanisms such as the blood-brain barrier (BBB). The direct transport route from the nasal cavity to the brain, bypassing the BBB, could potentially offer an exciting method for CNS drug delivery. The nose-to-brain (N2B) transport of therapeutic drugs is a scientifically well-known and clinically proven alternative route of administration as the so-administered drugs can bypass the BBB.

The therapeutic system within »N2B-patch«will consist of the active agent itself, a formulation containing the API, a hydrogel as carrier material for the formulation, and a suitable applicator (Figure 1). As the project coordinator, the Fraunhofer IGB is involved in the development of a medical therapy for the administration of active ingredients via the olfactory region in »N2B-patch«. 


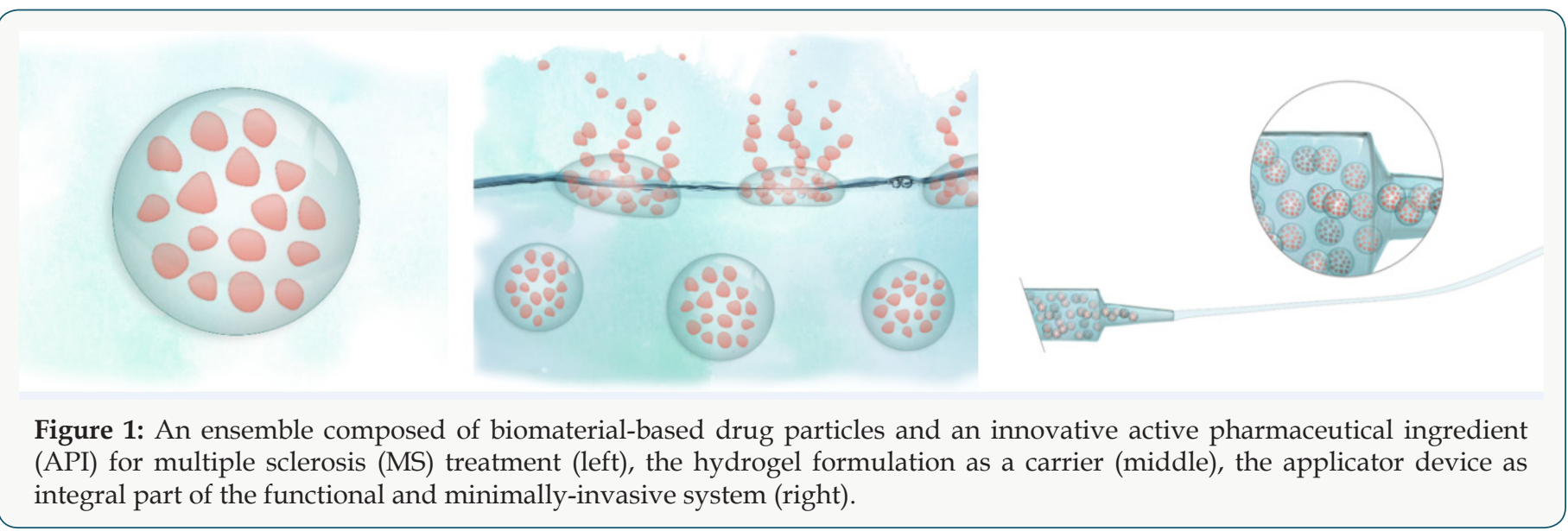

In the project the Fraunhofer IGB is concentrating on the formulation of the particles containing the active substance and the embedding of these particles into hydrogels. Various particle technologies are used to formulate micro- and nanoparticles loaded with APIs (Figure 2). Biobased or biodegradable polymers are chosen as the base material for the particles. Spray drying is a suitable process to formulate different classes of molecules like

small organic compounds or biomolecules and spray drying is one method that can be used for an effective encapsulation of biomolecules like insulin or interferon without losing the bioactivity of the encapsulated protein [3]. Two- and three-fluid nozzles are used for the particle preparation. For the one-step preparation of core-shell particles by spray drying the three-fluid nozzle has already been successfully integrated into the process.
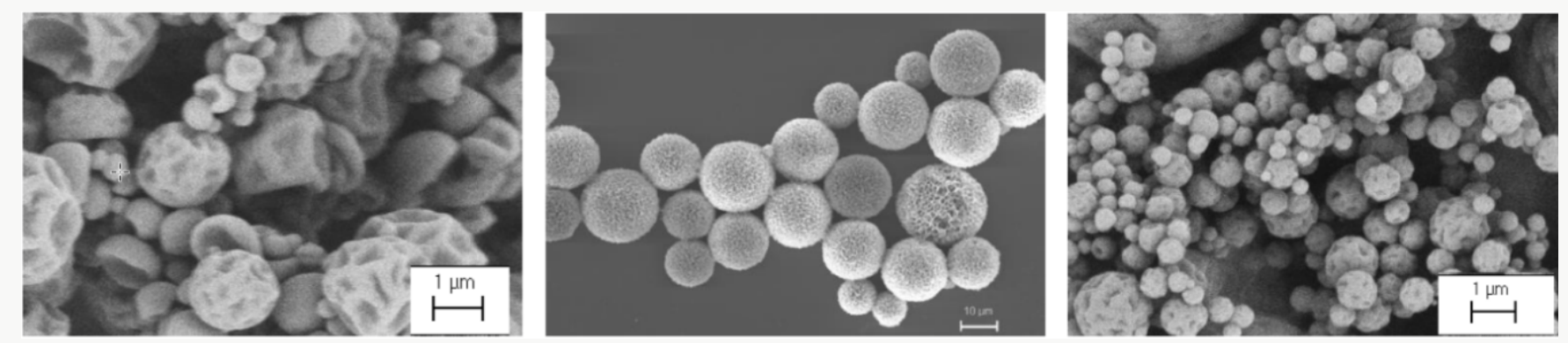

Figure 2: Scanning electron microscopic (SEM) images of different particles: Protein-loaded Insulin particles (left), proteinloaded chitosan particles (right), both prepared by a spray drying process and PEG-PLGA particles prepared by emulsion technologies (middle).

Various biobased polymers like chitosan, gelatine, alginate and different cross-linkers (covalent and non-covalent) in the production of particles are currently being tested. The biopolymer chitosan, known to interact with the mucosal surface, will be used as a mucoadhesive particle material or as mucoadhesive coating. Chitosan can, like other cationic molecules, interact with the mucus surface. The release properties of the APIs and particles can further be optimized by varying the particle material, the cross-linking degree or by the additional coating of the particles. The developed API-loaded particles can then be integrated into a hydrogel matrix and administered directly to the olfactory cleft with the new intranasal application system. The technology developed by the "N2B-patch « project may also be suitable for the use of different pharmaceutical ingredients. The envisaged direct drug delivery platform may also support the treatment of other demyelinating disorders and other CNS indications, e.g. stroke, neurodegenerative diseases or tumours.

\section{Acknowledgment}

This work has received funding from the European Union's Horizon 2020 research and innovation programme under grant agreement No 721098 - »N2B-patch«. The project started on 1st January 2017 with a duration of 48 months. More information can be found on the project website www.n2b-patch.eu.

\section{References}

1. (2007) Neurological Disorders: Public health challenges, World Health Organization.

2. (2008) Atlas multiple sclerosis resources in the world 2008. WHO Library Cataloguing-in-Publication Data.

3. Gruber-Traub C, Burger-Kentischer A, Gretzinger S, Hirth T, Weber A (2012) Chemie Ingenieur Technik 84: 343-348. 
(C) (P) This work is licensed under Creative

Submission Link: $\quad$ Submit Article

DOI: $10.32474 /$ DDIPIJ.2018.01.000116

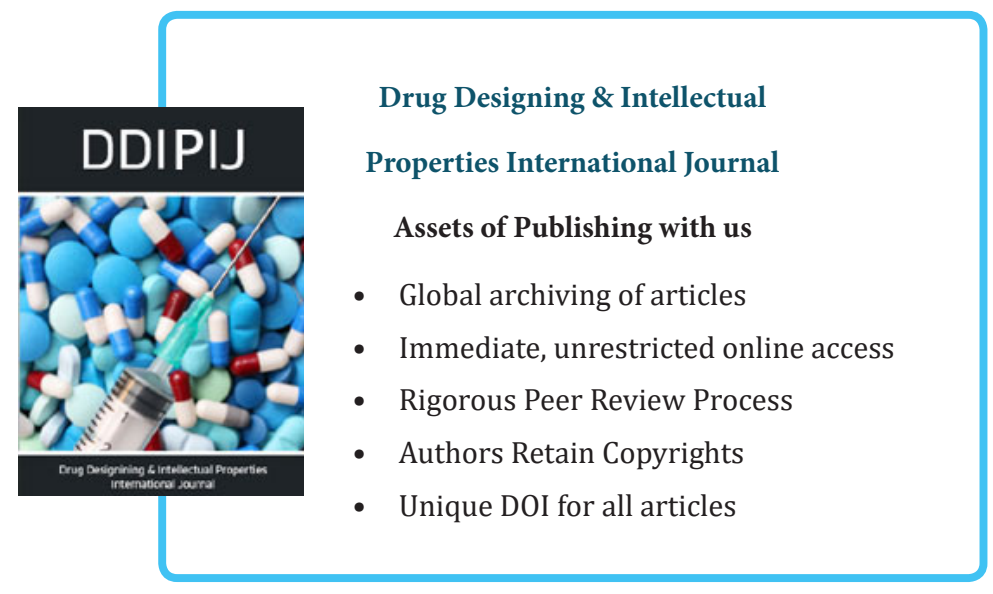

